\title{
Recall/recognition differences as a function of elementary school grade*
}

\author{
MELVIN H. MARX \\ University of Missouri, Columbia, Mo. 65201
}

Elementary school children were tested for retention of the identity of words whose spelling had been experimentally practiced 3 months earlier. In each of two schools, free recall scores were reliably greater for the lower grades and recognition scores reliably greater for the higher grades. These opposite functions, suggesting separate recall and recognition processes in memory, may also be interpreted as a result of the increasing use of language at the higher grades and the consequently greater interference with retrieval cues but concomitant greater ease in utilizing recognition cues.

\section{PROBLEM}

The typical experiment on memory involves a retention test on materials that have been acquired in a specifically planned training session. Seldom are materials which have been acquired in the pursuit of some other objective been tested for retention under carefully controlled conditions. The procedure for testing incidental learning lies somewhere in between these two extremes, because in that case, a learning and retention intent is usually explicit, albeit with regard to different items.

This paper reports some results from retention tests introduced with no advance warming 3 months after acquisition of spelling by elementary school children. Recall and recognition tests of the content of the learned materials (i.e., the identity of the specific words that had been used in the spelling practice) were introduced, in addition to a spelling retention test itself, when there had been no indication in training that the former type of test would be given at any time.

Free recall and recognition scores were compared over three elementary school grades in each of two schools,

* This research, reported at the November 1971 m e e t i g s of t he Psychonomic Society in St. Louis, Missouri, was supported in part by NSF Grant GB-8506, by Research Career Award 1-K6-MH-22,023 from the National Institute of Mental Health, and by a grant from the University of Missouri Research Council. I wish to thank Kathleen Marx for her assistance in the collection and processing of the data; thanks are also due John Mueller and David Witter for assistance in the statistical analysis. I am indebted to Dr. Thomas Southard, Superintendent of the Pinellas County, Florida, Public Schools, and his successor, Mr. Nicholas Mangin; to Mr. Ralph Hinkle, Principal, and to the following teachers of the Azalea Elementary School: Mrs. Spangler, Mrs. McFarland, Miss Utter, Mrs. Chamberlain, Mrs. Fannin, and Mrs. Charles; and to Mrs. Lillian Laird, Principal, and the following teachers of the Lynch Elementary School: Miss Privette, Mrs. Tibbetts, Miss List, Mrs. McMullen, Mrs. Parker, and Miss Reynolds. with different manipulations of independent variables. It was believed that data such as these might provide useful hints in the comparison of these two memory-testing procedures to supplement the more commonly used method.

\section{METHOD \\ Subjects}

The Ss were 158 children ( 73 boys, 85 girls) from two classes each in the second, fourth, and sixth grades in Experiment 1, and 167 children (81 boys, 86 girls) from two classes each in the fourth, fifth, and sixth grades of a second school in Experiment 2. All of the children in attendance at the time of training and testing were used from each class, so that the experimental Ss represented an unselected sampling from the school populations.

$$
\text { Apparatus }
$$

An Eastman Kodak Ektagraphic slide projector presented correct spellings of the words on a screen. Procedure: Experiment 1

Word selection. Each of the six teachers was provided a list of 30 words, selected for each grade from the words listed in the spelling manual (Kottmeyer \& Claus, 1968) for the next higher grade. They were asked to test their classes on the spelling of these words. The five words in each list that were least frequently spelled correctly were then used in the experiment. For the fourth and sixth grades, these words were supplemented by an additional set of five words selected for apparent difficulty from lists in the $S t$. Petersburg Times (1971) spelling-bee pamphlet. Because second-grade pupils were just beginning to print and spell words, it was decided that the original list of five words would be sufficient for them. All words used had four letters (e.g., warm, poor, hair), five letters (e.g., sugar, pious, query), or six letters (e.g., scheme, ballot, shriek) for the second, fourth, and sixth grades, respectively.
Familiarization session. The next week after the word screening, the two Es appeared briefly in each class to explain the general nature of the research and enable the children to become accustomed to their presence in the classroom.

Training sessions. Following an initial pretest, on which the children in each class were asked to spell each of the words on the list ( 5 for Grade 2, 10 for Grades 4 and 6 ), four training trials were administered with a test trial after each training trial. Each class was divided into two training groups, making a total of four groups per grade, and the following training operations were used: (1) Copy: S spelled each word as it was shown on the screen. (2) Remember: S watched the word as it was shown on the screen and spelled it from immediate memory as soon as the word disappeared from the screen. (3) Guess: S spelled each word as it was called out, then checked his spelling as the word was shown on the screen. (4) Think: S spelled the word to himself as it was called out, then checked the spelling as it was shown on the screen.

Operations copy and remember were administered in one class, guess and think in the other class.

Approximately $5 \mathrm{sec}$ were required for the oral cueing of each word and $10 \mathrm{sec}$ for both the task operation during training trials and the spelling effort during test trials. Brief definitions were added during the pretest trial and the first training trial. The correctly spelled words were shown on the screen for 5 sec. The order of the words was varied from trial to trial, with three random orders rotated over successive training and test trials, to prevent serial conditioning effects.

Retention session. After 3 months, without any advance notice to teachers or pupils, the retention tests were given.

In free recall, given first, Ss were asked to write down as many of the words which they had practiced earlier as they could remember. Two minutes were allowed.

In recognition, given next, all of the words actually practiced were now called out, mixed randomly with an equal number of similar words which had not been practiced. The distractor words for Grade 2 were drawn from the spelling manual, and those for Grades 4 and 6 were drawn from the spelling-bee pamphlet. Ss were asked to write down "yes" or "no," depending upon whether or not they thought the word called out was a previously practiced one. Each word was pronounced twice, and $5 \mathrm{sec}$ were allowed for responding on the test sheets. 


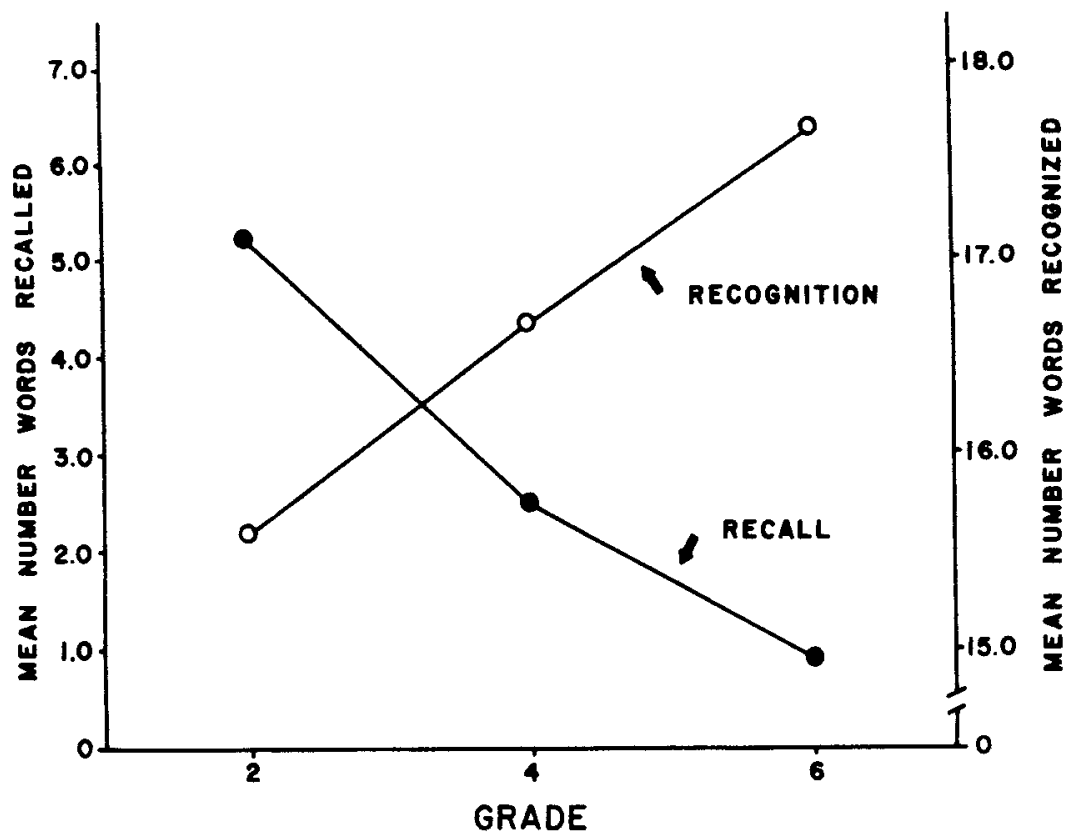

Fig. 1. Mean numbers of words recalled and recognized as a function of grade (Experiment 1).

Procedure: Experiment 2

Word selection. The words whose spelling was to be learned were all selected from lists in the St. Petersburg Times (1971) spelling-bee pamphlet. Six-letter words were used for the fourth (e.g., harass, mosaic, heifer) and fifth (e.g., fracas, coerce, hiatus) grades, and longer words (e.g., farcical, accolade, liaison) for the sixth grade.

Training session. One class from each grade was assigned to an individual practice condition and the other to a social practice condition. Pupils in each class were sorted into pairs, like-sex and of approximately the same ability level (as judged by the teacher). Ss in the individual condition worked by themselves in both training and test, whereas $S s$ in the social condition were permitted to cooperate quietly on spelling during training but worked by themselves in tests. In addition, each $\mathrm{S}$ performed (attempted to spell) on half of the words and observed (watched his partner perform) on the other half.

Following a pretest on the set of 12 words, four training trials were given, and then a test trial was administered. On training trials, each word was called out (with brief definitions during the pretest trial and the first training trial), and then $10 \mathrm{sec}$ were allowed for the performer to attempt to spell it. After six words, the correct spellings for these six words were shown by slide and the $O$ in each pair corrected errors; 2 min were allowed. Then the two Ss in each pair changed roles, and the last six words were used under the same procedure. The words
( $F<1.00$ for the former, $F=2.72$, $\mathrm{p}>.05, \mathrm{df}=2,134$ for the latter) for number of words correctly spelled. Retention differences can be considered to be substantially independent of any differential training effects, insofar as these are measured by correct spelling.

Figure 1 shows the recall and recognition curves as a function of grade level. The clearly opposite functions were in each case statistically reliable: for recall, $F=$ $54.47, \mathrm{p}<.01, \mathrm{df}=2,134 ;$ for recognition, $F=3.83, p<.05, \mathrm{df}=$ 2,134 . Neither of the other main effects (experimental treatment and sex) showed reliable differences.

These analyses were performed separately because of the difficulty of including a within-S factor in the unequal-Ns ANOVA. An additional analysis was therefore performed to include both the recall and the recognition measure, with the random elimination of $11 \mathrm{Ss}$ from the original 158. The means were nearly identical with those depicted in Fig. 1 (maximum deviation less than $2 \%$ ). Grade and retention measures interacted quite reliably, $F=42.92$, $\mathrm{p}<.01, \mathrm{df}=2,144$.

The recognition scores consisted of total number of correct responses, including acceptance of old words and Since each S made either a "yes" or single measure takes account of both false positives and false negatives.

In order to provide comparable data, all of the Grade 2 scores were rejection of new (distractor) words. "no" response for each word, this
In training, neither the experimental treatment nor the grade variable produced reliable differences reordered on successive trials.

retention session. Durin the same tests were administered as in Experiment 1.

\section{RESULTS \\ Experiment}

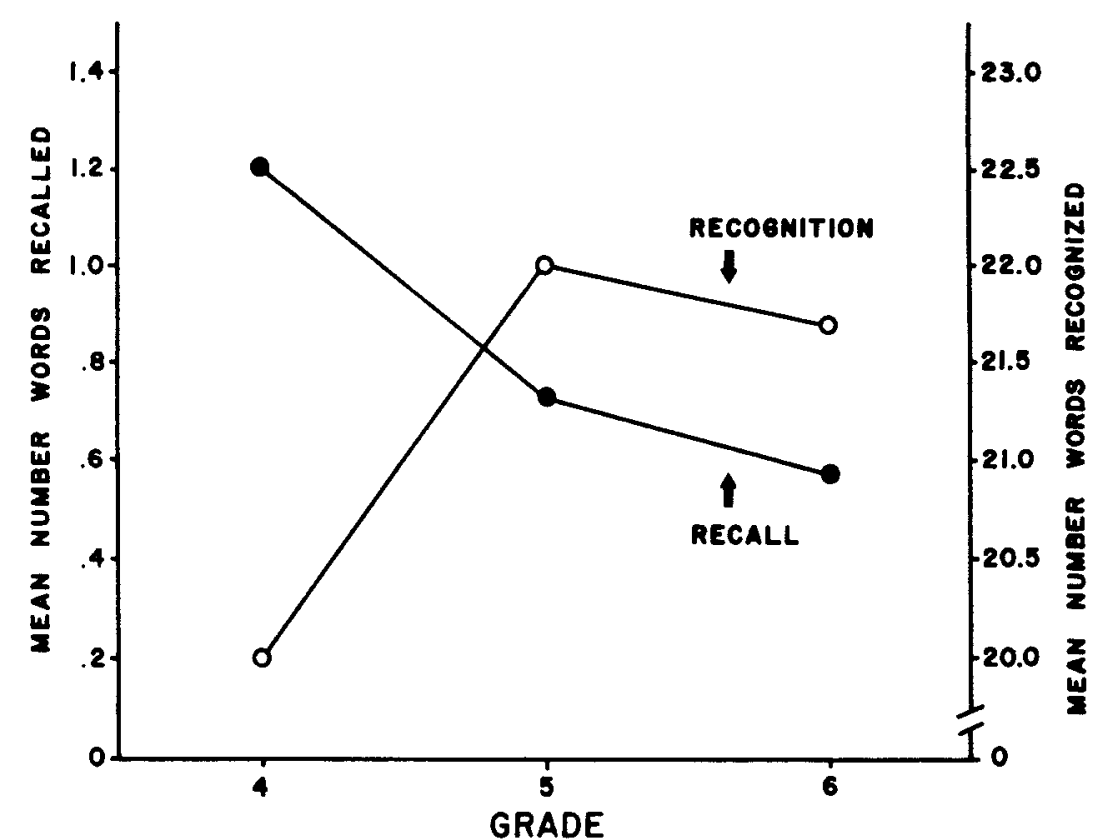

Fig. 2. Mean numbers of words recalled and recognized as a function of grade (Experiment 2). 
arbitrarily doubled, because only 5 words were used in that grade, whereas 10 words were used in the other two grades; since this operation applied to both types of retention measure, it should not have any bearing on their comparison.

\section{Experiment 2}

In training, both the grade and the sex main effects gave reliable differences: for grade level, $\mathrm{F}=4.97$, $\mathrm{p}<.01, \mathrm{df}=2,155$; for sex, $\mathrm{F}=5.57$, $\mathrm{p}<.05$, df $=1,155$. The mean test scores were as follows: Grade 4, 6.06; Grade 5, 5.47; Grade 6, 7.63; girls, 7.03 ; boys, 5.65 .

Figure 2 shows the recall and recognition curves as functions of grade level. It is apparent that the results of Experiment 1 were essentially replicated, with the lower grade again showing more recall but less recognition. The main effect of grade level was again statistically reliable in both cases: recall, $F=3.06$, $\mathrm{p}<.05$, df $=2,155$; recognition, $\mathrm{F}=$ $8.61, \mathrm{p}<.01, \mathrm{df}=2,155$.

Neither of the other main effects was statistically reliable for the recall measure, but for recognition, the main effect of sex was reliable ( $F=4.52$, $\mathrm{p}<.05, \mathrm{df}=1,155)$ : girls recognized more words than did boys (means of 21.70 and 20.93).

An additional analysis performed to test the interaction of grade and retention measure, as in Experiment 1, again indicated a high level of reliability, $\mathrm{F}=9.66, \mathrm{p}<.01, \mathrm{df}=$ 2,141 .

\section{DISCUSSION}

The main thrust of these results is reasonably apparent; they obviously suggest that recall and recognition, here shown to be opposite functions of grade level in two separate experimental situations, do in fact represent substantially different memory processes. They may most simply be interpreted, therefore, as additional evidence for the view that recall and recognition require separate theoretical accounts because they are differentially sensitive to a number of independent variables (see Kintsch, 1970 , pp. 277-282, for a recent summary of such cases).

An alternative account, however, is suggested by the direction of the differences, with lower grade levels showing more recall but less recognition for the same acquisition. This account would focus on differential experience with verbal materials as a critical differentiating factor. The higher grades, with such increasing amounts of verbal components in their school work, would not only be expected to suffer more interference in the retrieval of recall cues but would, at the same time and for the same reason, be able to identify more effectively the words earlier practiced in the recognition test, requiring fewer cues. Such an interpretation is consistent with the view that recall and recognition do not involve fundamentally different storage processes but, rather, function under varying requirements of learning, with recall demanding the higher level and recognition operating under partial degrees (cf. McNulty, 1965; Bruce \& Cofer, 1967), and also with the demonstration (e.g., Postman \& Stark, 1969) that associative interference is more effective on recall than on recognition. Increased verbal sophistication in the present type of retention test comparison might thus be expected to operate in the different directions simultaneously.

In view of the somewhat unorthodox methodology used in this study, special comment is indicated on certain points. One such point concerns the relationship between training scores and retention scores. This is not at all as critical a matter as it would normally be in retention experimentation, because in the present case, there was no very direct relationship between the two kinds of measure (i.e., number of words correctly spelled for the training measure and number correctly recalled or recognized for the retention measure, with correct spelling not required). Furthermore, no reliable training difference occurred over grades in Experiment 1, and the grade effect that was reliable in Experiment 2 was not linear, although Grade 6 was superior. In any case, the occurrence of opposite retention functions for the two types of measure insures that the retention results cannot simply be interpreted as a possible artifact of some inadequately measured training difference.

Even if a training effect could be shown to be correlated positively with one or the other of the two retention measures, there would still remain to be explained the negative relationship with the other measure. Thus, while information on training might well be helpful in suggesting the direction of interpretation of these results, lack of more directly relevant training data cannot be reasonably held to invalidate the observed opposition of retention trends over grades.

Similarly, the possible bias introduced by the use of a smaller number of items for the Grade 2 Ss in Experiment 1, and by the use of different words over grades, can hardly be used to explain the opposite trends in retention. Moreover, the fact that the directly opposite functions occurred in both experiments, with a great diversity of procedural details reduces the potential importance of any such bias.

The present results are seemingly at variance with certain others in the research literature. Shapiro \& Moely (1971), for example, have recently reported the expected increment in recall scores over elementary school grades, and Bach \& Underwood (1970) have reported a somewhat surprising superiority in recognition performance of Grades 2-6. It should be obvious, however, that there are far too many procedural variations among these experiments for any comparisons to be taken very seriously. Moreover, one last important methodological point should be noted. Since, in the present research, there was, as noted above, no direct manipulation or measure of the degree of acquisition of the identity of the words themselves, as contrasted with their spelling, there was no way to estimate the amount of such acquisition for the various groups, as would normally be done in experiments on memory. However, control of the acquisition variable was accomplished by the use of the same Ss for both of the retention measures. The present design may thus be regarded as less amenable to meaningful comparisons of one grade with another for either type of retention test alone-where large numbers of uncontrolled variables may operate-than to comparisons of the one retention measure with the other, as shown in the figures.

It is concluded that the present double retention test, which is relatively simple to perform and can be applied in a wide variety of learning situations, does provide useful data on retention complementary to the type normally gathered. Moreover, this type of testing permits a convenient measure of the interaction between type of retention test and other independent variables, such as grade level in the present instance.

\section{REFERENCES}

BACH, M. J., \& UNDERWOOD, B. J. Developmental changes in memory attributes. Journal of Educational Psychology, 1970,61, 292-296.

BRUCE, D., \& COFER, C. H. An examination of recognition and free recal as measures of acquisition and long-term retention. Journal of Experimental Psychology, 1967, 75, 283-289.

KINTSCH, W. Learning, memory and conceptual processes. New York: Wiley, 1970.

KOTTMEYER, W., \& CLAUS, A. Basic goals in spelling. New York: McGraw-Hill, 1968 .

McNULTY, J. A. An analysis of recall and recognition processes in verbal learning. Journal of Verbal Learning \& Verbal Behavior, 1965, 4, 430-436

POSTMAN, L., \& STARK, $K$. The role of response availability in transfer and interference. Journal of Experimental Psychology, 1969, 79, 168-177.

SHAPIRO, S. I., \& MOELY, B. E. Free recall, subjective organization, and learning to learn at three age levels. Psychonomic Science, 1971, 23, 189-191.

ST. PETERSBURG (Florida) TIMES. 17th Annual Suncoast Spelling Bee, 1971 . 